\title{
Research Progress of Precast Concrete Sandwich Panels
}

\section{Cong Zhao*, Miao Jikui}

School of Architecture and Urban Planning, Shandong Jianzhu University, Jinan, China

\section{Email address:}

501867489@qq.com (Cong Zhao),88149303@qq.com (Miao Jikui)

*Corresponding author

\section{To cite this article:}

Cong Zhao, Miao Jikui. Research Progress of Precast Concrete Sandwich Panels. Science Discovery. Vol. 7, No. 5, 2019 , pp. 354-358. doi: 10.11648/j.sd.20190705.26

Received: November 4, 2019; Accepted: November 27, 2019; Published: December 3, 2019

\begin{abstract}
The wallboard is an important support and guarantee for the development of prefabricated buildings, and it is also the main development direction of green wall materials in China during the 13th five-year plan period. Precast concrete sandwich panels is composed of inner and outer leaf concrete board, sandwich insulation layer and connecting parts, which has good performance of fire prevention, heat preservation and heat insulation. On the basis of introducing the types and characteristics of precast concrete sandwich panels, this paper summarizes the research status of precast concrete sandwich panels at home and abroad, analyzes the existing problems of sandwich panels research, and prospects the research direction of precast concrete sandwich panels.
\end{abstract}

Keywords: Precast Concrete, Sandwich Insulation, Wallboard

\section{预制混凝土夹芯板的研究进展}

\author{
从晊*，苗纪奎 \\ 山东建筑大学建筑城规学院, 济南, 中国 \\ 邮箱 \\ 501867489@qq.com（丛晊），88149303@qq.com（苗纪奎）
}

摘要：墙板部品化是发展装配式建筑的重要支撑和保障, 也是十三五期间我国绿色墙材的主要发展方向。预制混凝土 夹芯板由内外叶混凝土板、夹芯保温层和连接件三个部分组成, 具有良好的防火、保温隔热等性能, 是实现墙板部品 化, 推动装配式建筑发展的重要技术支撑。本文在介绍预制混凝土夹芯板的类型及特点的基础上, 总结了预制混凝土 夹芯板的国内外研究现状，分析了夹芯板研究存在的问题，并对预制混凝土夹芯板的研究方向进行了展望。

关键词: 预制混凝土, 夹芯保温, 墙板

\section{1. 引言}

目前我国正大力推动建筑行业的工业化发展, 发展装 配式建筑是实现建筑工业化的重要途径, 且装配式与传统 建筑模式相比具有明显优势。外墙是建筑物的主要围护结 构, 是建筑材料更新与建筑节能的重要研究领域, 其装配 化程度也在建筑工业化中起到至关重要的作用。
现有用于装配式的外墙主要分三种: 轻质条板、骨架 复合板、预制混凝土夹芯板。轻质条板为单一材料墙板, 自重轻, 但保温效果较差, 无法满足寒冷和严寒地区建筑 节能的要求; 骨架复合板自重轻, 抗震性能好, 机械化程 度高, 但价格偏高且骨架部位热桥问题严重, 局部热量损 失明显 [1]; 预制混凝土夹芯板可预制生产，且具有良好的 耐久性、保温隔热性能和防火性能等优点, 通过调整保温 层的厚度可满足北方地区的建筑节能要求。本文介绍了预 
制混凝土夹芯板的类型及特点, 综述了国内外研究现状, 并分析了国内外夹芯板存在的问题, 对预制混凝土夹芯板 的发展趋势进行了展望。

\section{2. 预制混凝土夹芯板的类型}

预制混凝土夹芯板是一种工业化生产的建筑墙板, 其 主要由内外叶混凝土墙板、夹芯保温层和连接件三个部分 组成。夹芯保温层材料通常为有机保温类保温板, 如聚苯 乙烯板、硬泡聚氨酯板和酚醛泡沫板等。美国预制混凝土 协会 (PCI) 将预制混凝土夹芯板按照复合方式分为三类: 非复合式、复合式、半复合式。

\section{1. 非复合式墙板}

非复合式墙板（图1a）是最早出现的，这种墙板使用 垂直于保温板平面设置的柔性连接件将保温层和内外叶 混凝土板连接在一起, 内外叶板独立作用, 共同承担水平 荷载, 外叶板较薄为非结构层, 内叶板较厚为结构层, 承 受重力荷载, 因此该种墙板的抗剪和抗弯能力小。

\section{2. 复合式墙板}

复合式墙板 (图1b) 使用连续的混凝土肋或钢筋组成 的桁架将保温层和内外叶混凝土板连接在一起, 混凝土肋 或钢筋即为连接件, 使内外叶板作为一个整体共同受力。 复合式墙板有较强的抗剪和抗弯能力, 可抵抗较大的侧向 力, 具有与相同厚度的传统墙板同样的强度和刚度。但混 凝土肋和钢筋传热系数较高, 使保温性能降低, 这种连接 方式也会阻碍内外叶层因温度变化而产生的热膨胀, 使墙 板出现热弯曲, 导致墙板间密封胶条开裂。

\section{3. 半复合式墙板}

半复合式墙板 (图1c) 是将保温层沿板长方向非连续 布置, 非连续处与内外叶板浇筑为一体, 非连续处一般位 于板的上下两端和中部, 保温层处仍由柔性连接件连接。 半复合式墙板的强度和刚度介于非复合式和复合式墙板 之间, 屈服强度与复合式墙板几乎相同, 但极限承载力稍 低, 非连续处易形成热桥, 与复合式墙板一样会出现热弯 曲。

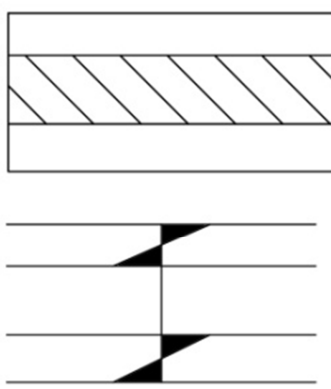

应力

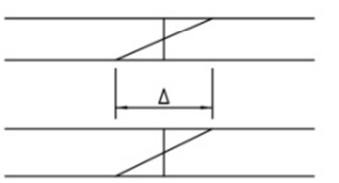

应变

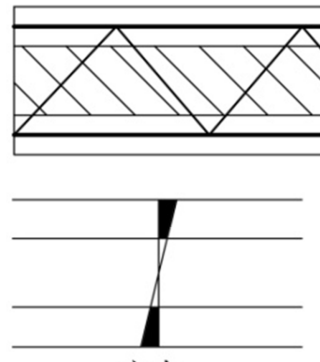

应力 b

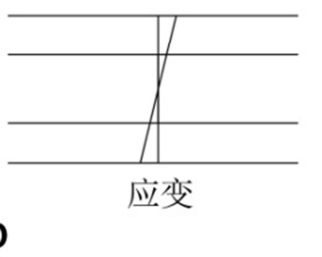

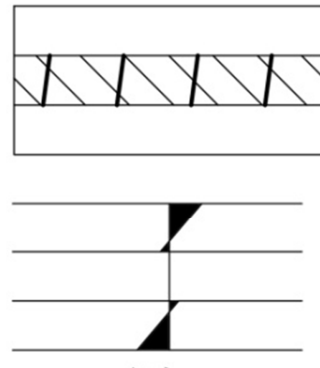

应力
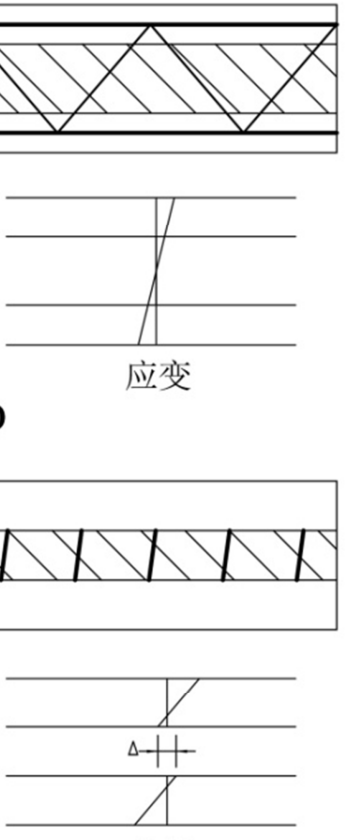

C

应变

图1 预制混凝土夹芯板的三种类型 $[2,3]$

\section{3. 预制混凝土夹芯板的研究现状}

\section{1. 国外研究现状}

夹芯系统最初在航空航天领域有较大突破, 1960年之 后开始在汽车产业、船舶业和建筑业广泛应用, 美国建筑 行业使用夹芯板已有 50 多年历史, 建筑行业所使用的预制 混凝土夹芯板是由工业化建筑系统（IBS）方法生产的实 心板改进而来, 由于其具有普通预制混凝土墙板的所有优 良特性和更优越的热工性能而得到推广。各国也相继颁布 实施了各项标准, 日本于1996年颁布实施预制混凝土夹芯 保温外墙工程应用技术标准, 该标准阐述了预制混凝土夹 芯保温外墙的设计、生产制作及施工验收以及性能要求, 作为预制混凝土夹芯保温外墙应用的技术指南一直沿用 至今；1997年美国建筑统一规范（UBC97）对夹芯墙中的 墙筛、有效厚度、有效面积、抗弯刚度及侧向支撑等都进 行了严格和详尽的规定, 在此基础上国外学者也对其做了 大量研究。

夹芯板虽然热工性能较好, 但使用连接件将各层连接 在一起的方法会产生热桥, 大大降低夹芯板的保温隔热作 用。1985年, 根据McCall [4]的研究, 在某些情况下, 夹 芯板连接部位所产生的热桥可使其热工性能降低多达 40\%。20世纪90年代, 美国 David C. Salmon、Amin Einea $[3,5]$ 等人对混凝土夹芯板进行了试验研究, 分析了板的变 形、极限强度及内外叶混凝土板之间剪力的传递等问题, 并采用导热系数小、抗拉强度高的纤维增强塑料FRP代替 钢筋, 使墙板保温性能更加良好, 同时在相关研究的基础 上建立起了混凝土夹芯板的理论体系。2013年Woltman G., Tomlinson D. [6]等对FRP连接件与传统钢和聚合物连接 连接件进行了对比, 试验证明FRP连接件的抗剪强度明显 
高于聚合物连接件, 但低于钢连接件, 并且随着保温层厚 度的增加, 连接件的强度会降低。2014年Paul HOPKINS [7] 等对由离散型、节段型和连续型FRP连接件组成的6块夹 芯板和2块实心板进行了试验, 结果表明使用FRP连接件 的板比实心板轻 $40 \%$, 并且不同类型的FRP连接件会影响 夹芯板的强度和刚度。这些研究表明使用FRP连接件代替 钢连接件可以有效避免热桥, 提高夹芯板的热工性能, 但 FRP连接件强度不如传统钢筋。

除了改变连接件的材料, 部分研究者通过提出新的设 计方法改善夹芯板的热工性能。2005年Byoung-Jun Lee和 Stephen Pessiki [8]在总厚度为 $279.4 \mathrm{~mm}$ 的三层预制混凝 土夹芯保温板中引入了热路径法, 该板有三层混凝土层和 两层保温层, 连续混凝土之间的实心混凝土部分在位置上 交错排列, 使其没有直接贯通板的连接部分, 与普通夹芯 板相比其热工性能提高 $19.8 \%$ 。然而, 这种墙板由于板材 厚度过大, 重量增加, 生产方法繁琐, 生产时间较长, 成 本较高, 不利于大规模使用。为了改善这一问题, 2018 年Bida [9]在 $150 \mathrm{~mm}$ 厚的双层超绝缘预制混凝土结构夹芯 板中使用了热路径的方法, 采用热箱试验法对连接件的三 种不同交错间距进行了试验, 并用有限元模型进行了验证。 尽管结构层使用了传统的钢筋和混凝土, 但与连接件未交 错布置相比，200毫米交错间距的热性能提高了 $117 \%, 300$ 毫米间距的热性能提高了 $207 \%, 400$ 毫米间距的热性能提 高了 $236 \%$ 。

此外, Rodrigo Lameiras [10]等人在2013年提出一种由 钢纤维增强自密实混凝土 (SFRSCC) 作为结构层的夹芯 板 (图2), 并用离散或连续的单向GFRP连接件连接, 该 新型板也有效减少了热桥所产生的的热量损失并减轻了 夹芯板的重量。

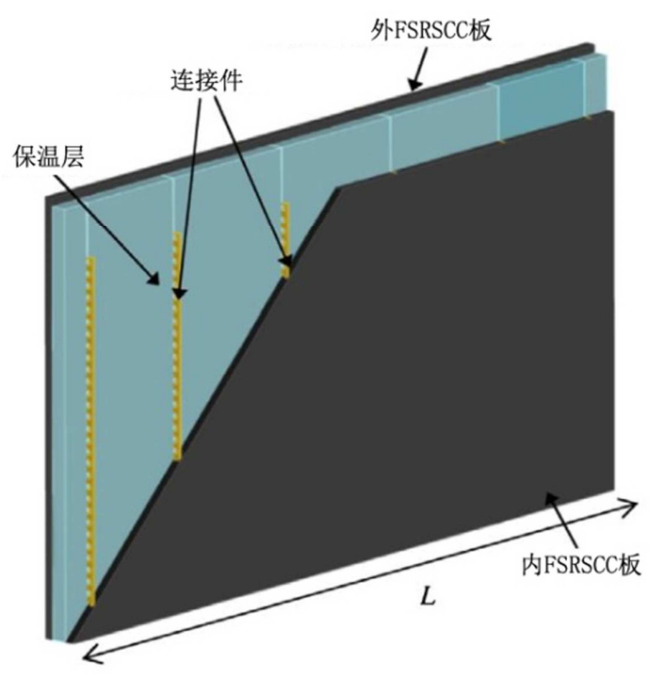

图2 钢纤维增强自密实混凝土 (SFRSCC) 夹芯板 [10]

在板的复合性能方面, 2011年Frank1 BA [11]的研究表 明使用GFRP连接件或实心混凝土连接可使预制混凝土夹 芯板的的复合作用百分比值接近 $100 \%$ ；2015年Kim J 和 You Y-C [12]对采用EPS保温板和XPS保温板的夹芯板进 行了复合程度的试验, 试验表明采用EPS保温板的预制混 凝土夹芯板比采用XPS保温板的具有更强的复合效果;
2017年Huanzhi Jiang [13]等对预制混凝土夹芯板使用不同 数量和分布方式的 $\mathrm{w}$ 型钢玻璃纤维增强聚合物 (SGFRP) 连接件进行了试验, 结果表明相比夹芯板中部, 板端部三 分之一处的连接件数量对板的复合作用影响更大。

综上所述, 国外对预制混凝土夹芯板的研究已较为成 熟, 对夹芯板的热工、力学以及复合性能方面都有大量研 究, 并在此基础上进一步研发了众多性能优良的新型预制 混凝土夹芯板。

\section{2. 国内研究现状}

国内对预制混凝土夹芯板的研究于上世纪 90 年代前 后开始, 众多学者对预制混凝土夹芯板做了大量试验分析, 虽然起步较晚, 但发展至今也有较大进展。

1986年北京市建筑工程研究所的叶大藏[14]对混凝土 岩棉复合外墙板的类型进行了总结并介绍了其构造做法, 提出可以用岩棉复合板取代当时常用的浮石混凝土板。同 年, 屠仲元 [15]对薄壁混凝土岩棉复合外墙板进行了研究, 叙述了其结构力学性能、材料性能、热工性能等, 表明了 这种复合墙板的节能效果。但国内早期使用的夹芯板存在 制作工序复杂、自重过大施工困难等问题, 不利于推广。

1999年烟台大学的林子臣[16-17]对钢丝网架夹芯板 的物理与力学性能进行了研究, 经试验证明, 钢丝网架夹 芯板具有施工速度快、质轻、优良的热工、隔声性能及一 定的耐火性和抗冻性等优点。2010年哈尔滨工业大学的王 振龙 [18]同样利用ANSYS对钢筋网架-混凝土组合夹芯板 作非线性分析, 对该板的抗弯性和抗压性能进行研究, 研 究表明该板具有良好的抗弯承载能力、抗压性能及保温性 能。这些研究成果为今后此种板材的相关试验与分析奠定 了基础。

在夹芯板热工性能方面, 部分学者对夹芯板的连接件 也进行了大量研究。2013年同济大学的薛伟辰、杨佳林 [19] 等人对夹芯板的连接件进行了研究, 结果表明, 采用 FRP 连接件的预制夹芯保温墙体的传热系数满足国家建筑节 能标准要求。但FRP连接件在我国使用成本过高, 不利于 市场应用推广。2016年东南大学刘卉[20]提出一种预制混 凝土夹芯保温板的新型连接件形式, 即在 $\mathrm{W}$ 型钢筋外包一 层玻璃纤维制成钢筋玻璃纤维复合筋（图3），利用玻璃 纤维的低导热系数减少纯金属件的热桥问题, 同时改善纯 玻璃纤维筋抗剪能力差的问题。

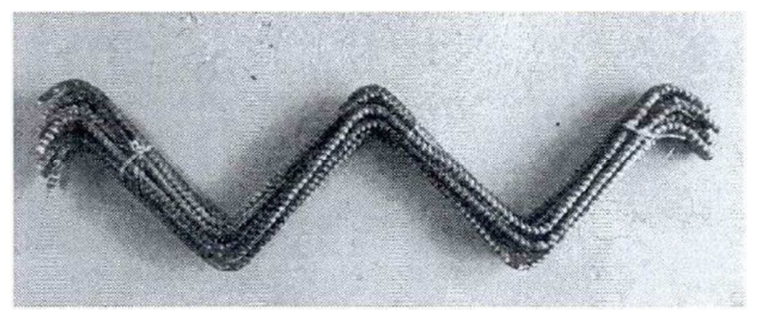

图3 新型连接件

我国还对预制混凝土夹芯板的设计方法进行了大量 研究, 以解决连接方式造成的热量损失。2001年王士风[21] 提出一种新型钢筋混凝土组合网架夹芯板（图4），简称 WZ板, 该板是将平面钢筋网架的两层弦杆浇筑在内外叶 
层中, 又在两层网架混凝土中填充保温芯板, 使夹芯板有 较大承载力且无需设置钢筋混凝土边框, 而且没有了热桥。 2017年王玲、孙俊蕾[22]等提出在夹芯保温墙板的桁架钢 筋连接件周围填充聚苯颗粒保温砂浆, 以提高墙板的热工 性能, 并结合热桥分析软件 PTemp 对比分析桁架钢筋周 围填充不同材料的保温效果, 结果表明此墙板采用填充的 聚苯颗粒保温砂浆能有效弥补钢筋导热系数大的缺点。 2017年鲍鹏[23]等采用对聚苯乙烯泡沫塑料保温板挖洞, 再在两边浇筑陶粒混凝土内外叶层的方法, 使内外叶层通 过开洞处的混凝土连接在一起, 制成新型预制混凝土夹芯 板, 这种新型墙板提高了墙板的整体受力性能并减小了由 于连接而产生的热桥。

除此之外, 还有研究者通过使用新型材料提高预制混 凝土夹芯板的性能。2018年申亚楠[24]通过调整结构层混 凝土配合比, 选用泡沫玻璃板和FPR连接件制备出一种预 制夹心保温复合墙板，对墙板进行了试生产，并采用不同 外墙体系进行了建筑能耗模拟分析以及做出墙板适用性 综合评价。

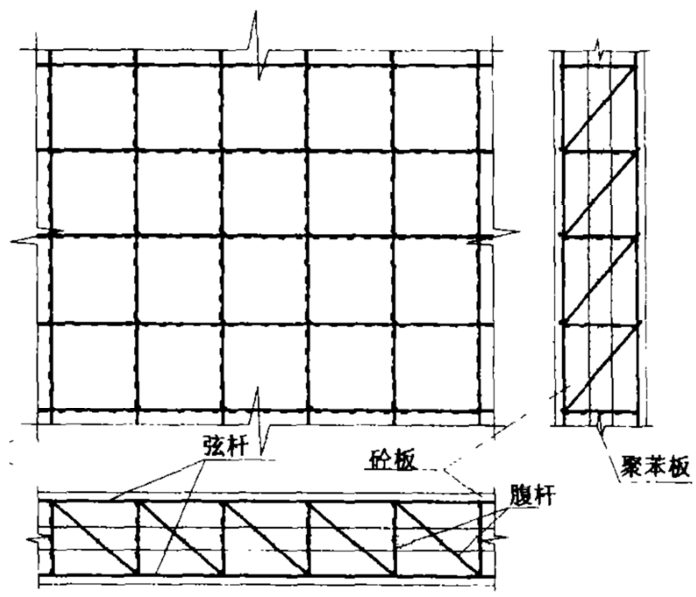

图4 WZ板构造图[22]

国内对预制混凝土夹芯板的研究主要集中在力学及 热工性能方面, 为此也设计研发了一批新型预制混凝土夹 芯板, 并将新型材料应用于夹芯板以提高板的性能, 但在 板的复合性能方面研究较少。

\section{3. 预制混凝土夹芯板存在的问题}

从目前的国内外研究来看, 针对预制混凝土夹芯板的 研究主要集中在夹芯板的力学性能和解决连接件引起的 热量损失问题上, 但目前研究还存在以下问题:

(1) 国内新型预制混凝土夹芯板种类繁多, 设计方 法多样, 但对新型夹芯板的研究主要集中在力学和热工性 能上, 且主要停留在理论研究, 对墙板应用技术研究较少, 缺少可大量应用于实际工程的夹芯板。

（2）预制混凝土夹芯板大多以传统混凝土作为结构 层, 传统混凝土密度大, 重量大, 不利于建筑节能以及装 配式建筑的墙板安装。国内外开始使用轻质墙体材料, 如 泡沫混凝土、加气混凝土、钢纤维增强自密实混凝土来替
代传统混凝土, 但这些轻质材料还存在密度低, 力学性能 和耐久性较差等问题。

(3) 国内目前使用较多的为普通钢筋连接件, 但导 热系数较高, 在夹芯板中易形成热桥; 近年来, 国内外开 始研究使用的纤维塑料 (FRP) 连接件导热系数更低, 然 而FRP连接件抗剪强度不如钢连接件, 且比钢筋连接件造 价高, 不利于市场应用推广, 除此之外, 在夹芯板中使用 FRP材料还存在粘性滑移、破坏应变低、弹性模量低、脆 性大、易老化等缺点。

\section{4. 结论与展望}

预制混凝土夹芯板具有保温隔热、防火、耐久性能好 等优点, 能够最大限度地实现墙板部品化, 是推进我国装 配式建筑发展的重要技术支撑, 但其自身还存在一定的不 足, 国内对夹芯板的研究也尚不够完善。因此, 对预制混 凝土夹芯板的研究可从以下三个方面展开:

（1）探索新型的墙板设计方法, 优化墙板的构造, 并对新型墙板应用技术进行研究, 研发热工性能优良、质 轻高强、造价低廉、施工便捷的新型夹芯板。

（2）选择合适的内外叶板替代材料, 用质轻、热工 性能好、强度高、吸水率低的材料来替代传统混凝土以降 低墙板自重, 同时满足内外叶板的力学与耐久性能要求。

(3) 研发新型的内外叶板连接件, 在满足力学性能 优良、造价低廉的要求下，同时解决夹芯板采用传统钢筋 连接件存在的热桥问题, 从而提高墙板的经济性、热工和 力学性能。

\section{参考文献}

[1] 陈璐. 竹筋一陶粒混凝土粘结性能及复合墙板抗弯性能的 试验研究与理论分析[D].山东大学,2012:10-11.

[2] 肖力光, 许晓晛. 预制混凝土夹芯墙板的研究与应用 [J].吉林 建筑工程学院学报,2014,31(03):15-18.

[3] Salmon D C, Einea A, Tadros M K, et al. Full scale testing of precast concrete sandwich panels [J]. Aci Structural Journal, 1997, 94 (4): 354-362.

[4] Mccall W C. Thermal properties of sandwich panels[J]. Concrete International, 1985, 7 (1): 35-41.

[5] Einea A, Salmon D C, Fogarasi G J, et al. State-of-the-Art of Precast Concrete Sandwich Panels [J]. Pci Journal, 1991, 36 (6): 78-98.

[6] Woltman G, Tomlinson D, Fam A. Investigation of Various GFRP Shear Connectors for Insulated Precast Concrete Sandwich Wall Panels [J]. Journal of Composites for Construction, 2013, 17 (5): 711-721.

[7] Paul HOPKINS, Tom NORRIS, Nicolas PEÑA, An CHEN. Development of FRP Plate Shear Connector for Insulated Concrete Sandwich Panels. International Institute for FRP in Construction. The 7 th International Conference on FRP Composites in Civil Engineering, 2014: 8. 
[8] Lee B J, Pessiki S. Thermal performance evaluation of precast concrete three-wythe sandwich wall panels [J]. Energy \& Buildings, 2006, 38 (8): 1006-1014.

[9] Bida, S. M., Aziz F. N. A. A., Jaafar M. S., Hejazi F., Nabilah A. B. Thermal performance of super-insulated precast concrete structural sandwich panels [ENB 176C (2018) 418430] [J]. Energy and Buildings, 2019, 186.

[10] Lameiras R, Barros J, Valente I B, et al. Development of sandwich panels combining fibre reinforced concrete layers and fibre reinforced polymer connectors. Part I: Conception and pull-out tests [J]. Composite Structures, 2013, 105: 446-459.

[11] Frankl B A, Lucier G W, Hassan T K, et al. Behavior of precast, prestressed concrete sandwich wall panels reinforced with CFRP shear grid [J]. Pci Journal, 2011, 56 (2): 42-54.

[12] Kim J H, You Y C. Composite Behavior of a Novel Insulated Concrete Sandwich Wall Panel Reinforced with GFRP Shear Grids: Effects of Insulation Types [J]. Materials, 2015, 8 (3): 899-913.

[13] Huanzhi Jiang, Zhengxing Guo, Jiabin Liu.Composite Behavior of Sandwich Panels with W-Shaped SGFRP Connectors [J]. KSCE Journal of Civil Engineering (2018) 22 (5): 1889-1899.

[14] 叶大藏.混凝土岩棉复合外墙板的构造及生产工艺 [J].建筑 技术, 1986(04):2-6.

[15] 屠仲元, 冯立南.薄壁混凝土岩棉复合外墙板的研究与试制 [J].混凝土与水泥制品,1986(03):51-55.
[16] 林子臣.钢丝网架水泥聚苯乙烯夹芯板的物理性能 [J].建筑 结构,1998(09):52.

[17] 林子臣.钢丝网架水泥夹芯板冲击性能实验研究 [J].建筑技 术开发,1999(05):30-31.

[18] 王振龙. 钢筋网架-混凝土组合夹芯墙板性能研究 [D].哈尔 滨工业大学,2010:64.

[19] 杨佳林,薛伟辰,栗新.预制夹芯保温墙体FRP连接件的力学 性能试验 [J]. 江苏大学学报 (自然科学 版),2013,34(06):723-729.

[20] 刘卉. 预制混凝土夹芯保温外挂墙板研究 [D]. 东南大 学,2016:81-82.

[21] 郎涁,王士风.一种新型的节能住宅建筑体系—WZ体系 $[\mathrm{J}]$. 青岛建筑工程学院学报,2005(02):1-5.

[22] 王玲,孙俊蕾,付素娟. 新型夹心保温墙板温度效应的数值模 拟分析[J].建筑科学,2017,33(12):103-109.

[23] 鲍鹏,马少春,韩学行,钟山,付吴,岳建伟.陶粒混凝土夹芯保 温复合墙板性能分析 [J]. 河南大学学报 (自然科学 版),2017,47(05):591-596.

[24] 申亚楠. 预制夹心保温复合墙板的研制及适用性评价[D]. 中国矿业大学,2018:81-82. 